\title{
Alterações na Composição Corporal Decorrentes de um Treinamento de Musculação em Portadores de Síndrome de Down
}

\author{
Body Compostion Alterations Resulting From Weight Training in \\ Subjects With Down Syndrome
}

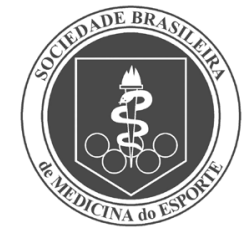

Artigo Original

\begin{abstract}
José Florentino Neto
Luciano Meireles de Pontes ${ }^{2,3}$

José Fernandes Filho $4,5,6$

1. Mestrando em Ciência da Motricidade Humana - Programa de Pós-graduação Stricto Sensu em Ciência da Motricidade Humana. Universidade Castelo Branco (UCB). Rio de Janeiro, RJ, Brasil.

2. Programa de Pós-graduação em Saúde da Criança e do Adolescente. Universidade Federal de Pernambuco (UFPE). Recife, PE, Brasil.

3. Laboratório de Avaliação Física Unipê/Sanny. João Pessoa, PB, Brasil. 4. Docente do Departamento de Educação Física. Escola de Educação Física e Desporto. Universidade Federal do Rio de Janeiro (UFRJ). Rio de Janeiro, RJ, Brasil.

5. Pesquisador do Conselho Nacional de Desenvolvimento Científico e Tecnológico (CNPq) - nível 1D. 6. Laboratório de Biociências da Motricidade Humana - Labimh (RJ).
\end{abstract}

Endereço para correspondência: Laboratório de Pesquisa Corpore Scientific.

Rua Telegrafista Deusdedit José de Carvalho, 33 - Bessa

58037-248 - João Pessoa, PB.

E-mail: zecacorpore@hotmail.com

\begin{abstract}
RESUMO
O objetivo deste estudo foi analisar as alterações na composição corporal decorrentes de um treinamento de musculação em portadores da síndrome de Down. Métodos: Participaram 15 portadores da síndrome de Down (22,1 \pm 7,5 anos), divididos em dois grupos: experimental (G1 = oito) submetidos a um programa de treinamento de musculação e controle (G2 = sete) sem intervenção. A composição corporal foi estimada através da equação de sete dobras cutâneas. As avaliações foram realizadas em pré e pós-teste, com frequência semanal de três vezes em dias alternados e duração de 60 minutos. 0 protocolo experimental foi elaborado com nove exercícios sob a forma de circuito, sendo realizados em três séries de oito a 12 repetições, com intervalos de descanso entre 30 e 60 segundos. Resultados: Foi observada no G1 diminuição significativa no percentual de gordura $(-2,0 \% ; p=0,036)$ e massa gorda $(M G)(-1,4 \mathrm{~kg} ; p=0,000)$. Os controles apresentaram aumento desfavorável no $\% G(+1,0 \% ; p=0,043)$ e $M G(+2,0 \mathrm{~kg}, p=0,004)$. Em relação à massa magra (MM), observou-se ganho significativo no G1 (+1,2kg; $p=0,008)$; tendo o G2 apresentado redução de MM (-0,8kg; $p=0,003)$. Conclusão: $O$ treinamento de musculação apresentou efeito favorável, promovendo redução na gordura corporal e aumento na MM, podendo ser sugerido para indivíduos com características similares às dos aqui investigados.
\end{abstract}

Palavras-chave: trissomia, levantamento de peso, antropometria.

\begin{abstract}
The aim of this study was to examine changes in body composition resulting from weight training among individuals with Down Syndrome. Material and methods: 15 subjects with Down Syndrome (22.1 \pm 7.5years) participated in the study and were divided in two groups: experimental group (G1 $=08$ ) submitted to a weight training program, and a control group $(G 2=07)$, without any intervention. Body composition was estimated through the equation of seven skinfolds. Pre- and post-test evaluations were performed three times every other day and had duration of 60 minutes. The experimental protocol consisted in nine rounds of exercise, performed in three sets of 8 to 12 repetitions and rest intervals of 30-60 seconds in-between. Results: Significant decrease of fat percentage was found in G1 $(-2.0 \%, p=0.036)$ and absolute fat $(-1.4 \mathrm{~kg} ; \mathrm{p}$ $=0.000)$. The controls presented unfavorable increase in fat percentage $(+1.0 \%, p=0.043)$ and absolute fat $(+0.8 \mathrm{~kg}, \mathrm{p}=0.004)$. Regarding lean mass $(\mathrm{LM})$, significant increase in $\mathrm{Gl}(+1.2 \mathrm{~kg}, \mathrm{p}=0.008)$, and decrease in $G 2(-2.0 \mathrm{~kg} ; p=0.003)$ was observed. Conclusion: The weight training program had a favorable effect, promoting body fat reduction and muscle mass increase, suggesting hence that this program should be indicated for individuals with similar characteristics to in this investigation.
\end{abstract}

Keyword: trisomy, weight lifting, anthropometry.

\section{INTRODUÇÂO}

Recentemente, vem ocorrendo um crescente interesse no tocante ao estudo das particularidades dos portadores de necessidades especiais. Dentro desse enfoque considera-se a síndrome de Down ou trissomia 21, uma condição genética reconhecida há mais de um século, constituindo uma das causas mais frequentes de deficiência mental, compreendendo cerca de 18\% do total de deficientes mentais em instituições especializadas e sendo, dessa forma, uma das mais comuns entre todas as síndromes que geram má-formação nos seres humanos ${ }^{(1)}$.
Os portadores de síndrome de Down compõem um grupo específico onde se observam prevalências de sobrepeso e obesidade superiores às verificadas em populações adultas consideradas saudáveis. Conforme dados reportados na literatura epidemiológica, cerca de 45,0\% dos homens e 56,0\% das mulheres com síndrome de Down apresentam excesso ponderal quando classificados pelo índice de massa corpórea ${ }^{(2)}$.

Alguns aspectos bem peculiares no estilo de vida de portadores de síndrome de Down ${ }^{(3)}$ estão relacionados com o sedentarismo, o uso de fármacos e os hábitos alimentares desequilibrados, sabendo-se que esses determinantes podem levar ao aparecimento de diversas patologias e/ou distúrbios, dos quais se destaca a obesidade(4). 
Nos últimos anos, estudos ${ }^{(5,6)}$ vêm procurando enfocar levantamentos na tentativa de fornecer subsídios que possam servir de linha de base na análise de atributos associados ao estado nutricional e composição corporal de portadores da síndrome de Down.

A análise dos compartimentos da composição corporal em qualquer fase da vida pode refletir o status de saúde como também o nível de aptidão funcional. Desse modo, observa-se que estudos ${ }^{(7,8)}$ têm ressaltado a importância do treinamento de força. ou popularmente "musculação", na melhora da composição corporal nas diversas idades.

Sobre esse foco, pesquisadores ${ }^{(9)}$ destacam o incremento de atividades físicas e esportivas, como a prática de exercícios de musculação, na melhora efetiva da composição corporal. Apesar disso, alguns estudiosos ${ }^{(10)}$ vêm encontrando em seus trabalhos resultados contrários em relação ao impacto da musculação na composição corporal. Atrelado a tais achados, especula-se que algumas limitações nos procedimentos metodológicos, como a falta de supervisão e intensidade no incremento das cargas de treino e a desconsideração do princípio da individualidade biológica, possam influenciar negativamente no binômio "musculação e composição corporal".

Considerando que parte dos estudos disponíveis na literatura médica e epidemiológica focaliza os efeitos de exercícios não sistematizados ou com implementos não convencionais, como elásticos, bolas de borracha ou mesmo sugerindo a realização de treinamento físico no próprio domicílio(10) na melhora da composição corporal, justifica-se a produção de novos estudos que abordem o treinamento físico sistematizado sob a supervisão de profissionais da Ciência do Esporte, sendo essa proposta sugerida e recomendada por organizações internacionais como o American College of Sport Medicine ${ }^{(11)}$. Dessa forma, conhecendo a escassez de publicações nacionais relativas ao treinamento de musculação com portadores de síndrome de Down, acredita-se que ainda seja pertinente a produção de um experimento enfatizando a prescrição e acompanhamento da prática de exercício de musculação nessa população, por observar que uma lacuna de conhecimento ainda necessita ser consolidada, corroborando as informações já conhecidas sobre os benefícios dessa atividade para a saúde, em que podem ser mencionadas a segurança na execução dos movimentos e as condições de monitoramento individual. Portanto, o objetivo deste trabalho foi verificar as alterações na composição corporal decorrentes de um treinamento de musculação em portadores da síndrome de Down.

\section{MÉTODOS}

A presente pesquisa enquadra um desenho experimental devido à ação de intervenção que foi realizada em um dos grupos e apresenta característica de séries de casos e caráter comparativo em dois momentos (pré e pós-teste). A partir de uma lista de 18 inscritos no programa de atividades físicas para portadores de necessidades especiais, optou-se para este estudo em excluir três indivíduos por apresentarem diagnóstico de cardiopatia.

\section{Amostra}

Participaram 15 portadores de síndrome de Down (22,1 \pm 7,5 anos), lotados em um Centro de Atividades Especiais (CAE) localizado na cidade de João Pessoa (PB). Todos os indivíduos foram submetidos a uma avaliação médica, obtendo um diagnóstico favorável para a prática de exercícios físicos com pesos antes de ser distribuídos aleatoriamente em dois grupos: experimental (G1) e controle (G2). O G1 foi composto por oito indivíduos, sendo seis do sexo masculino e dois do feminino, que realizaram o treinamento de musculação. O G2 foi formado por sete indivíduos, sendo cinco do sexo masculino e dois do feminino, que realizaram suas atividades habituais durante o mesmo período de intervenção.

\section{Variáveis e instrumentos da pesquisa}

A determinação da massa corporal (MC) foi realizada através de balança mecânica modelo Filizola (Indústria Filizola S/A, Brasil), com acuidade de $100 \mathrm{~g}$ e capacidade de até $150 \mathrm{~kg}$. A determinação da estatura se fez por meio de altímetro acoplado à balança com escala em centímetros. Para estimativa da porcentagem de gordura (\%G) foi aplicado o protocolo de Sete Dobras Cutâneas (DC) proposto por Jackson Pollock ${ }^{(12)}$, utilizando um adipômetro científico da marca Sanny (American Medical do Brasil, São Paulo). O fracionamento da composição corporal foi realizado em dois componentes: massa gorda (MG) e massa magra (MM). A estimativa da MG foi realizada através do cálculo: $M G=M C * \% G / 100$. Para a MM optou-se pelo seguinte cálculo: MM = MC - MG. O material utilizado no treinamento foi constituído de um banco supino reto, três barras, um par de halteres de $1 \mathrm{~kg}, 2 \mathrm{~kg}$ e $3 \mathrm{~kg}$, dois colchonetes e 30 quilos de anilhas, todos da marca Physicus.

\section{Procedimentos do estudo}

Em um primeiro momento foi realizado contato com o CAE, onde foi explicada a razão da pesquisa e esclarecida a necessidade de se fazer o estudo com portadores de síndrome de Down. Após a autorização da instituição, foi realizada uma sistematização para a seleção aleatória dos participantes. Em seguida, os pais e/ou responsáveis foram convocados para uma reunião, onde foram explicados os objetivos, riscos e benefícios da pesquisa, seguindo a Lei 196/96, que envolve Pesquisas Experimentais com Seres Humanos ${ }^{(13)}$. Após a autorização por escrito do Termo de Consentimento Livre e Esclarecido foi agendada uma avaliação através de mensurações antropométricas, cuja etapa antecedeu o início do treinamento de musculação. Após o período de seguimento, realizou-se a avaliação final (pós-teste). O protocolo do estudo foi submetido ao Comitê de Ética em Pesquisa da Universidade Castelo Branco (RJ) e aprovado sob o no 26/2007.

\section{Protocolo experimental}

O programa de treinamento de força foi realizado por um período de 12 semanas, com três sessões semanais em dias alternados no CAE. Para tanto, foram utilizados os seguintes materiais: banco supino reto, três barras, um par de halteres de $1 \mathrm{~kg}, 2 \mathrm{~kg}$ e $3 \mathrm{~kg}$, dois colchonetes e $30 \mathrm{~kg}$ de anilhas, todos da marca Physicus. Os exercícios envolveram grandes grupos musculares, sendo o protocolo de treinamento baseado na proposta descrita por Le Clair e Elliot ${ }^{(14)}$, estando mais adequado para a população estudada. Os exercícios realizados incluiram: supino plano, agachamento, elevação lateral, flexão de joelho, remada unilateral, panturrilha em pé, rosca direta, abdominal supra e tríceps francês. O treinamento foi executado sob a forma de circuito com três séries de oito a 12 repetições em cada estação, com intervalos de descanso entre as séries de 30 a 60 segundos. Os treinos tiveram duração aproximada de 60 minutos. As cargas foram determinadas através de estimativas onde os sujeitos conseguissem realizar no mínimo oito e no máximo 12 repetições. Foi adotada a frequência de participação como critério de exclusão, não permanecendo aqueles que, por qualquer motivo, apresentassem três faltas consecutivas. Durante o período experimental não houve perda no G1; três sujeitos foram excluídos no G2 em razão da desistência na avaliação pós-teste. A pesquisa foi realizada no segundo semestre de 2008, com frequência geral superior a 75,0\%. Não houve reposição das sessões de treinamento para os voluntários faltosos.

\section{Análise dos dados}

O tratamento dos dados incluiu estatística descritiva de média, mínimo, máximo e desvio padrão (DP), e técnica inferencial para análise intra e intergrupos (G1/G2). Foi utilizado o teste de Shapiro-Wilk para verificar a normalidade na distribuição das variáveis contínuas, sendo 
empregados para comparação das médias os testes $t$ de Student pareado para amostras dependentes (análise intragrupos) e não pareado para grupos independentes (análise intergrupos). Adotou-se o nível de significância de 5\% $(p<0,05)$ para rejeição da hipótese de nulidade.

\section{RESULTADOS}

Os resultados apresentados na Tabela 1 demonstraram na variável MC que o grupo experimental não sofreu alteração significante no seu peso ( $p=0,825)$, condição diferente observada nos controles, que aumentaram significativamente no pós-teste $\left(+1,2 \mathrm{~kg} ; \mathrm{p}=0,040^{*}\right)$. Verificando a composição corporal, observou-se que o G1 apresentou diminuição significativa nos níveis de \%G $(-2,0 \% ; p=0,036)$ e $M G(-1,4 k g$; $p=0,000)$. Seus pares do $G 2$ obtiveram significância desfavorável nas mesmas variáveis $(+1,0 \%, p=0,043 ;+2,0 \mathrm{~kg}, p=0,004)$. Em relação à MM, observou-se aumento significante no G1 (+1,2kg; 0,008); tendo o $\mathrm{G} 2$ apresentado redução de $\mathrm{MM}(-0,8 \mathrm{~kg} ; \mathrm{p}=0,003)$.

Tabela 1. Composição corporal: comparação das médias e desvio padrão do pré e pós-teste intragrupos

\begin{tabular}{|c|c|c|c|}
\hline \multirow{2}{*}{$\frac{\text { Composição corporal }}{\text { G1 }}$} & \multicolumn{2}{|c|}{ Média \pm DP } & \multirow{2}{*}{$p$} \\
\hline & Pré-teste & Pós-teste & \\
\hline$M C(k g)$ & $63,0 \pm 8,4$ & $62,9 \pm 7,9$ & 0,825 \\
\hline Estatura (m) & $1,53 \pm 0,46$ & $1,54 \pm 0,46$ & 0,942 \\
\hline$\% G(\%)$ & $25,3 \pm 10,9$ & $23,3 \pm 12,3$ & $0,036^{*}$ \\
\hline$M G(k g)$ & $16,6 \pm 8,4$ & $15,2 \pm 9,1$ & $0,000^{*}$ \\
\hline MM (kg) & $46,4 \pm 4,3$ & $47,6 \pm 5,6$ & $0,008^{*}$ \\
\hline G2 & Pré-teste & Pós-teste & $p$ \\
\hline $\mathrm{MC}(\mathrm{kg})$ & $53,7 \pm 3,5$ & $54,9 \pm 3,3$ & $0,040^{*}$ \\
\hline Estatura (m) & $1,49 \pm 0,44$ & $1,49 \pm 0,44$ & 0,940 \\
\hline$\% G(\%)$ & $21,7 \pm 3,9$ & $22,7 \pm 3,8$ & $0,043^{*}$ \\
\hline MG (kg) & $11,7 \pm 6,2$ & $13,7 \pm 6,7$ & $0,004^{*}$ \\
\hline MM (kg) & $44,4 \pm 5,4$ & $43,6 \pm 5,7$ & $0,003^{*}$ \\
\hline
\end{tabular}

* $p<0,05$ (diferença significativa).

G1/G2 = grupo experimental/grupo controle;

$\mathrm{MC}=$ massa corporal; $\% \mathrm{G}=$ porcentagem de gordura; $\mathrm{MG}=$ massa gorda; $\mathrm{MM}=$ massa magra

A tabela 2 apresenta a comparação das médias, desvio padrão e significância estatística do pré e pós-teste intergrupos. Observa-se que o G2 apresentou MC superior ao G1 durante todo o experimento ( $p$ > 0,05). Em relação à estatura, os grupos mostraram perfil semelhante. 0 $\%$ G se apresentou no pré-teste significativamente superior nos sujeitos do G1 ( $p=0,049)$. No pós-teste não foi observada diferença entre os grupos ( $p=0,075$ ). Quando analisada a MG, o G1 iniciou o experimento com um valor médio estatisticamente maior no pré-teste $(p=0,049)$. Após as alterações ocorridas no período de intervenção, os grupos não mostraram diferenças significativas ( $p=0,447)$. Na análise da MM, os sujeitos iniciaram o estudo com valores semelhantes e, no pós-teste, valores estatisticamente diferentes $(p=0,049)$.

\section{DISCUSSÃO}

É observado na literatura epidemiológica que os portadores de síndrome de Down necessitam diminuir os níveis de gordura corporal, principalmente por considerar que o sobrepeso e a obesidade estão associados a males à saúde e qualidade de vida ${ }^{(15)}$. Nesse sentido, este estudo objetivou analisar as alterações na composição corporal decorrentes de um treinamento de musculação em portadores da trissomia 21 , sendo essa análise intra e intergrupos. Nesse contexto, optou-se por
Tabela 2. Composição corporal: comparação das médias e desvio padrão do pré e pós-teste intergrupos.

\begin{tabular}{c|c|c|c|c}
\hline \multirow{2}{*}{ Composição corporal } & \multicolumn{2}{|c}{ Média \pm DP } & \multirow{2}{*}{$\mathbf{p}$} \\
\cline { 3 - 4 } & G1 & G2 & \\
\hline \multirow{3}{*}{$M C(\mathrm{~kg})$} & Pré-teste & $63,0 \pm 8,4$ & $53,7 \pm 3,5$ & $0,022^{*}$ \\
\cline { 2 - 5 } & Pós-teste & $62,9 \pm 7,9$ & $54,9 \pm 3,3$ & $0,033^{*}$ \\
\hline \multirow{3}{*}{ Estatura $(\mathrm{m})$} & Pré-teste & $1,53 \pm 0,46$ & $1,49 \pm 0,44$ & 0,164 \\
\cline { 2 - 5 } & Pós-teste & $1,54 \pm 0,46$ & $1,49 \pm 0,44$ & 0,163 \\
\hline \multirow{3}{*}{$\% \mathrm{G}(\%)$} & Pré-teste & $25,3 \pm 10,9$ & $21,7 \pm 3,9$ & $0,049^{*}$ \\
\cline { 2 - 5 } & Pós-teste & $23,3 \pm 12,3$ & $22,7 \pm 3,8$ & 0,075 \\
\hline \multirow{2}{*}{$M G(\mathrm{~kg})$} & Pré-teste & $16,6 \pm 8,4$ & $11,7 \pm 6,2$ & $0,049^{*}$ \\
\cline { 2 - 5 } & Pós-teste & $15,2 \pm 9,1$ & $13,7 \pm 6,7$ & 0,447 \\
\hline \multirow{2}{*}{$M M(\mathrm{~kg})$} & Pré-teste & $46,4 \pm 4,3$ & $44,4 \pm 5,4$ & 0,168 \\
\cline { 2 - 5 } & Pós-teste & $47,6 \pm 5,6$ & $43,6 \pm 5,7$ & $0,049^{*}$ \\
\hline
\end{tabular}

${ }^{*} \mathrm{p}<0,05$ (diferença significativa)

$\mathrm{G} 1 / \mathrm{G} 2$ = grupo experimental/grupo controle;

$\mathrm{MC}=$ massa corporal; \%G = porcentagem de gordura; $\mathrm{MG}=$ massa gorda; $\mathrm{MM}=$ massa magra

um seguimento de treinamento de musculação devido à segurança propiciada na sua prática e por entender que esse tipo de treino físico contribui para o aumento da massa magra e elevação do metabolismo basal, aumentando o gasto calórico e, consequentemente, contribuindo para a redução da gordura corporal|(16).

O perfil antropométrico observado em ambos os grupos (G1/G2) enquadrou os participantes nos padrões já mensurados em outros estudos com portadores de síndrome de Down.

Em ensaio utilizando a análise de densitometria radiológica de dupla energia, Mendonça e Pereira ${ }^{(17)}$ estimaram a composição corporal de 10 portadores de síndrome de Down do sexo masculino. Os valores da massa corporal mensurados variaram entre $52,2 \mathrm{~kg}$ e $75,5 \mathrm{~kg}$ e estatura entre $146,5 \mathrm{~cm}$ e $170,0 \mathrm{~cm}$. A \% dos investigados foi de $25,8 \%$, semeIhante à média apresentada pelo G1 anteriormente à participação no programa de treinamento. Quando comparadas as médias verificadas no presente estudo, ambos os grupos apresentaram valores inseridos na amplitude observada no estudo referenciado ${ }^{(17)}$.

Um aspecto que deve ser enfatizado é que estudos sobre o perfil antropométrico e fracionamento da composição corporal em portadores de algum tipo de deficiência mental ainda são pouco referenciados, o que dificulta a comparação dos resultados deste trabalho. Acredita-se que isso pode estar relacionado com a maior dificuldade de realizar as técnicas de mensuração e a falta de protocolos de avaliação específicos.

Sobre a existência de estudos envolvendo desenhos do tipo experimental e pré-experimental com portadores de síndrome de Down, algumas pesquisas já foram publicadas, sendo estas fundamentais para a produção de novos ensaios científicos sobre o tema em questão.

Em um estudo de caso, Silva Jr. et al.(18) analisaram o aumento da resistência muscular localizada e os efeitos de um treinamento de musculação para um jovem com síndrome de Down. O protocolo de pesquisa contemplou apenas exercícios para os membros superiores, com três séries de 12 repetições com cargas moderadas e por um período de seis meses. Apesar de não ter havido modificações significativas ( $p>0,05)$, o treinamento promoveu, além de tendência para o aumento da resistência muscular, alterações positivas na composição corporal. Na presente pesquisa, onde os exercícios envolveram maior número de grupos musculares em seu protocolo, observaram-se resultados satisfatórios e significativos, corroborando a tendência encontrada no estudo de caso, levando-nos a perceber que o treinamento executado de forma sistemática e adaptado a cada situação proporciona benefícios relacionados com a saúde. 
Investigando a influência de um protocolo de exercício nos níveis de tecido adiposo, Ordonez et al. ${ }^{(19)}$ observaram 22 portadores de síndrome de Down, todos do sexo masculino, durante o período de 12 semanas. Antes da intervenção, 31,8\% estavam com sobrepeso e 27,3\% com obesidade e, após o treinamento, obtiveram redução significativa na $\% G$, passando de 31,8\% para 26,0\%, respectivamente. No presente estudo, os resultados também corroboram os descritos por Ordonez et al. ${ }^{(19)}$, considerando que o G1 apresentou alterações significativas no $\%$ G, MG e MM quando comparado com o G2, que concluiu o estudo com níveis de gordura superiores aos valores do pré-teste e valores inferiores na MM, condição de perda que expõe tais sujeitos à hipotonia muscular, o que já é uma característica marcante na síndrome de Down. Nesse contexto, as evidências obtidas nos resultados deste estudo são consistentes, no sentido de que se observaram redução efetiva da porcentagem de tecido adiposo e aumento de MM no grupo que sofreu a intervenção, sinalizando para a eficiência da musculação para melhora desse componente relacionado com a saúde.

Quando analisada a composição corporal intergrupos, percebeuse que o grupo experimental iniciou o estudo com nível superior de $\% G$. Com a intervenção sofrida, o G1 apresentou perda significante ( $p$ $<0,05)$, fazendo com que no pós-teste os grupos não apresentassem mais diferenças nessa variável, já que foi vista tendência inversa entre os grupos, com diminuição significante de gordura no G1 e aumento nos controles. Essa tendência interferiu na MG e MM, demonstrando a eficácia do protocolo experimental aplicado ao grupo que sofreu a intervenção.

Outros estudos que envolveram intervenções diferentes da proposta do presente estudo revelaram mudanças significativas na composição corporal e aptidão física de portadores de síndrome de Down ${ }^{(20-23)}$.
Algumas limitações desta pesquisa estão relacionadas com o próprio comprometimento mental que enquadra o portador de síndrome de Down como, por exemplo, baixa assimilação das informações dadas na fase de intervenção e falta de concentração durante o treinamento proposto. Desse modo, acredita-se que, apesar dos resultados significantes observados nos componentes da composição corporal, a presença de mais profissionais para supervisionar os exercícios colocados no protocolo experimental teria sido relevante para evitar a não realização na execução completa de determinados exercícios por alguns sujeitos durante as sessões de treinamento.

Assim, devido às limitações inerentes a estudos envolvendo populações especiais e considerando o tamanho amostral, os resultados devem ser vistos com cautela. Entretanto, os dados evidenciados mostram uma tendência que pode ser referenciada e comparada com outros estudos com desenho e população semelhantes.

\section{CONCLUSÃO}

A prática da musculação proposta nesta pesquisa apresentou impacto favorável, promovendo redução na gordura corporal e aumento na massa corporal magra, podendo ser sugerido para indivíduos com características semelhantes aos aqui investigados. Sugere-se que novos estudos sejam realizados, incluindo uma casuística de maior dimensão e realizando o monitoramento dietético, visando melhor compreensão dos efeitos das intervenções não farmacológicas sobre a composição corporal.

Todos os autores declararam não haver qualquer potencial conflito de interesses referente a este artigo.

\section{REFERÊNCIAS BIBLIOGRÁFICAS}

1. Moreira LMA, EL-Hani CN, Gusmão FAF. A síndrome de Down e sua patogênese: considerações sobre o determinismo genético. Rev Bras Psiquiatria 2000;22:96-9.

2. Rubin SS, Rimmer JH, Chicoine B, Braddock D, McGuire D. Overweight prevalence in persons with Down syndrome. Ment Retard 1998;36:175-81.

3. Prasher VP. Overweight and obesity among Down's syndrome adults. J Intellect Disabil Res1995;39:437-41.

4. Henderson A, Lynch SA, Wilkinson S, Hunter M. Adults with Down's syndrome: the prevalence of complications and health care in the community. Br J Gen Pract 2007;57(534):50-5.

5. Melville CA, Cooper SA, McGrother CW, Thorp CF, Collacott R. Obesity in adults with Down syndrome: a case control study. J Intellect Disabil Res 2005;49:125-33.

6. Husain MA. Body mass index for Saudi children with Down's syndrome. Acta Paediatrica 2003; 92:1482-5.

7. Carmeli E, Barchad S, Masharawi Y, Coleman R. Impact of a walking program in people with Down syndrome. J Strength Cond Res 2004;18:180-84.

8. Shefer G, Carmeli E, Rauner G, Yablonka-Reuveni Z, Benayahu D. Exercise running and tetracycline as means to enhance skeletal muscle stem cell performance after external fixation. J Cell Physiol 2008;215:265-75.

9. Richmond SR, Godard MP. The effects of varied rest periods between sets to failure using the bench press in recreationally trained men. J Strength Cond Res 2004;18:846-9.

10. Brasil RRLO, Conceição FL, Coelho CW, Rebello CV, Araújo CGS, Vaisman M. Efeitos do treinamento físico contra resistência sobre a composição corporal e a potência muscular em adultos deficientes de hormônio do crescimento. Arq Bras Endocrinol Metab [serial on the Internet]. 2001;45:134-40.

11. Pescatello LS, Franklin BA, Fagard R, Farquhar WB, Kelley GA, Ray CA. American College of Sports Medicine position stand. Exercise and hypertension. Med Sci Sports Exerc 2004;36:533-53.

12. Resende FAC, Rosado LEFPL, Priore SE, Franceschini. Aplicabilidade de equações na avaliação da composição corporal da população brasileira. Rev Nutr 2006;19:357-67.
13. Brasil, Ministério da Saúde. Conselho Nacional de Saúde. Manual operacional para comitês de ética em pesquisa. Brasília (DF): Ministério da Saúde, 2002.

14. Le Clair DA, Elliot D. Movement preparation and the costs and benefits associated with advance information for adults with Down syndrome. Adapt Phys Activ Q 1995;12:239-49.

15. Organização Pan-Americana de Saúde. Doenças crônico-degenerativas e obesidade: uma estratégia mundial sobre alimentação saudável, atividade física e saúde. Brasília (DF): Organização Pan-Americana de Saúde, 2003

16. Nam S, Lee S, Nan Y, Lee C, Choi K. The changes in muscle strength and body composition after 12 weeks of circuit weight training program. Proceedings of Symposium on Human-Environment System 2003:27:67-70

17. Mendonça GV, Pereira FD. Medidas de composição corporal em adultos portadores de síndrome de Down. Rev Bras Educ Fís Esp 2008;22:201-10.

18. Silva Jr. CA, Tonello MGM, Gorla Jl, Calegari DR. Musculação para um aluno com síndrome de Down e o aumento da resistência muscular localizada. Efdeportes 2007;104:1-7.

19. Ordonez FJ, Rosety M, Rosety-Rodriguez M. Influence of 12-week exercise training on fat mass percentage in adolescents with Down syndrome. Med Sci Monit 2006;12:416-9.

20. Carmeli E, Kessel S, Coleman R, Ayalon M. Effects of a treadmill walking program on muscle strength and balance in elderly people with Down's syndrome. J Gerontol A Biol Sci Med Sci 2002;57:M106-10.

21. Hernandez-Reif M, Ironson G, Field T, Largie S, Deigo M, Mora D, et al. Children with Down syndrome improved in motor function and muscle tone following massage therapy. Journal of Early Intervention 2006:176:395-410

22. Tsimaras VK, Fotiadou EG. Effect of training on the muscle strength and dynamic balance ability of adults with down syndrome. J Strength Cond Res 2004;18:343-7.

23. Lewis CL, Fragala-Pinkham MA. Effects of aerobic conditioning and strength training on a child with Down syndrome: a case study. Pediatr Phys Ther 2005;17: 30-6. 